\title{
Comparison of Marginal Fit of Metal Copings in Two Different States with and without Using Ring Liners: An In Vitro Study
}

\author{
Pankaj P Kadam¹, Rupali Patil ${ }^{2}$, Arti Gachake ${ }^{3}$, Vijaysinh More ${ }^{4}$, Harshwardhan Sawant ${ }^{5}$, Bhagyashree Kalsekar ${ }^{6}$
}

\begin{abstract}
Aim and objective: To compare the marginal fit of $\mathrm{Ni}-\mathrm{Cr}$ copings made by casting in two different states (dry and wet) with either cellulose ring liners or no ring liners.

Materials and methods: An in vitro study was conducted on 40 patterns which were invested, and a burnout casting procedure was used, using a Ni-Cr alloy to obtain the cast copings. The cast copings were divested, cleaned, and run through a finishing procedure. The copings were seated on the stainless steel die and microscopically evaluated for marginal discrepancies at two predetermined reference points using an optical microscope (Rieichert, Austria). Statistical analysis was done using Pearson's correlation coefficient and Chi-square test, keeping 95\% confidence intervals and having a $p$ value of less than 0.05 statistically significant.

Results: Our results identified marginal discrepancies in all the $\mathrm{Ni}-\mathrm{Cr}$ cast copings, which significantly differed from each other. The copings obtained from casting with a cellulose ring liner in a wet state showed a significantly higher value followed by casting using a cellulose ring liner in a dry state. The mean marginal discrepancy values were within the clinically acceptable range for all the $\mathrm{Ni}-\mathrm{Cr}$ cast copings included in two groups.

Conclusion: Our study concludes that the copings cast with a cellulose ring liner in dry states had the least amount of vertical marginal discrepancies as compared to those that were cast with a ring liner in two different forms or those cast without a ring liner, suggesting that the use of cellulose ring liner in a dry state is favorable for all casting procedures.

Clinical significance: Measuring gaps or discrepancies at margins is the commonly used method to determine the fit of Ni-Cr copings. In order to minimize marginal inaccuracies, various authors have suggested different methods to improve the marginal adaptation of cast restorations. There are compensation methods like setting expansion, hygroscopic expansion, and thermal expansion of the investment, which are used to assess metal shrinkage during cooling.

Keywords: Casting, Cellulose liners, Marginal fit, $\mathrm{Ni}-\mathrm{Cr}$ cast copings.

The Journal of Contemporary Dental Practice (2021): 10.5005/jp-journals-10024-3180
\end{abstract}

\section{INTRODUCTION}

Casting is a process that converts the wax pattern of a dental restoration into a replicate made of a dental alloy using centrifugal force and pressure or pressure and a vacuum with a mold cavity. ${ }^{1}$ The casting process is used to produce dental restorations such as inlays, onlays, crowns, bridges, and removable partial dentures. Because these castings must meet stringent dimensional requirements, the casting process is extremely demanding. In dentistry, virtually all castings are done using some form of adaptation of the lost-wax technique. ${ }^{2}$ The lost-wax technique has been used for centuries but was not common in dentistry until 1907 when WH Taggart introduced his technique with a casting machine. ${ }^{1}$

WH Taggart first described the lost-wax casting process in the context of dentistry and observed that the resulting restorations were undersized. The accuracy of the fit of a cast restoration is essential to its longevity because it reduces the amount of plaque accumulation in the marginal area, provides better mechanical properties (stability, resistance), uses less cement space (reduced likelihood of marginal leakage), and improves the aesthetic result. ${ }^{3}$

Precise marginal adaptation is necessary to improve mechanical, biological, and aesthetic prognoses. The marginal fit of the castings is largely dependent on careful tooth preparation, accurate

\begin{abstract}
${ }^{1-6}$ Department of Prosthodontics and Crown and Bridge, Bharati Vidyapeeth (Deemed to be University) Dental College and Hospital, Pune, Maharashtra, India

Corresponding Author: Pankaj P Kadam, Department of Prosthodontics and Crown and Bridge, Bharati Vidyapeeth (Deemed to be University) Dental College and Hospital, Pune, Maharashtra, India, Phone: +919881124147, e-mail: panks_619@yahoo.co.in

How to cite this article: Kadam PP, Patil R, Gachake A, et al. Comparison of Marginal Fit of Metal Copings in Two Different States with and without Using Ring Liners: An In Vitro Study. J Contemp Dent Pract 2021;22(11):1262-1267.
\end{abstract}

Source of support: Nil

Conflict of interest: None

impressions, and precision castings paired with careful finishing and cementation procedures. ${ }^{2,3}$

The marginal fit of cast restorations is one of the most researched subjects in fixed prosthodontics. Despite close attention to clinical and laboratory procedures, marginal discrepancies of cast restorations are inevitable. Previous reports show that the clinically acceptable marginal discrepancies for cast restorations range from 10 to $160 \mu \mathrm{m} .{ }^{4,5}$ However, there is a general consensus

(c) The Author(s). 2021 Open Access This article is distributed under the terms of the Creative Commons Attribution 4.0 International License (https://creativecommons. org/licenses/by-nc/4.0/), which permits unrestricted use, distribution, and non-commercial reproduction in any medium, provided you give appropriate credit to the original author(s) and the source, provide a link to the Creative Commons license, and indicate if changes were made. The Creative Commons Public Domain Dedication waiver (http://creativecommons.org/publicdomain/zero/1.0/) applies to the data made available in this article, unless otherwise stated. 
among authors that marginal discrepancies that exceed $100 \mu \mathrm{m}$ constitute an unacceptable marginal opening. A number of factors can be detrimental to the marginal adaptation of cast restorations, including clinical and technical errors by dentists or dental technicians concerning the geometry of the tooth preparation, the type of finish line, and the degree of the taper, and incompatibilities between dental materials, such as impression materials, waxes, die stones, and casting investments. ${ }^{6}$

Previously, asbestos liners were used to compensate for this expansion. However, while these liners were in use for many years, they have since been abandoned due to their association with carcinogenesis and were replaced with paper ceramic liners. ${ }^{7,8}$ Whenever a solid metal ring or a casting flask is used for casting, provisions must be made to permit the investment to expand. If this provision is not made, the mold may actually become smaller due to reverse pressure resulting from the confined expansion of the setting. Asbestos liners were previously the preferred material but no longer used due to their carcinogenic potential-asbestosis/ mesothelioma. ${ }^{1}$ Aluminosilicate ceramic liners are fibrous ceramic aluminum silicate as alternative to asbestos liners. The liner increases the degree of normal setting expansion in the investment and also increases the amount of water absorption from the investment during the setting, which leads to semi-hygroscopic expansion., ${ }^{9,10}$

Several previous studies compared the previously described casting techniques for fixed restorations. Keyf and Erman ${ }^{1}$ evaluated marginal discrepancies using three different casting ring liners (no liners, wet liners, and dry liners). They used cellulose liners as the wet liner and ceramic liners as the dry liner.

Cellulose liners can be used dry or prewetted. In the past, cellulose ring liners were not used in both a dry and prewetted state within the same study. ${ }^{11}$ Therefore, we aimed to measure the marginal gaps and to determine the clinical acceptability of single-unit castings in phosphate-bonded investments that were cast with either dry cellulose ring liners, wet cellulose ring liners, or no casting ring liners. This study aimed to assess, compare, and determine the vertical marginal accuracy of lost-wax castings with dry cellulose ring liners, wet cellulose ring liners, and no casting ring liners. The primary objectives of this study were to compare casting techniques using different casting ring liners and determine which of the liners produce single full coverage restorations with a superior marginal fit and to determine if the casting ring liners that are used during the casting technique can be routinely used to fabricate cast restorations using the metal ring casting technique. The secondary objective was to provide dentists, patients, and dental laboratory technicians with time-saving, cost-effective and accurate techniques to fabricate clinically acceptable single-unit full coverage restorations.

\section{Materials and Methods}

This is an in vitro study conducted after obtaining relevant permissions from the Institutional Review Board in Pune. The study was performed following the guidelines of in vitro studies: Checklist for Reporting In vitro Study (CRIS) guidelines. ${ }^{12}$

\section{Study Armamentarium and Materials}

The study armamentarium used was metal die, counter die, casting ring, crucible, digital electronic thermometer, vacuum mixing machine, wax burnout furnace, induction casting machine, sandblasting machine, and digital optical microscope. The materials used were acrylic tray material, Exaflex polyvinyl siloxane impression material, regular, ultra-rock type IV gypsum, die hardener, crown and bridge modeling wax, Sprue wax, debubblizer, casting ring liner, phosphate-bonded investment material, $\mathrm{Ni}-\mathrm{Cr}$ dental casting alloy.

\section{Methodology}

A steel die fabrication was done using a stainless steel die assembly (Fig. 1). It was used to fabricate the standardized wax patterns. The master die simulated a crown preparation with a $6^{\circ}$ total axial wall taper. The axial height of the die and its occlusal diameter was $6 \mathrm{~mm}$ and the finish line was a $90^{\circ}$ shoulder, $1 \mathrm{~mm}$ in width. Occlusal crosshairs were placed for precise wax pattern repositioning. The die could be accurately positioned in a stainless steel former (counter die) that had an opening in the center $1 \mathrm{~mm}$ larger than the die in all dimensions. This was followed by a stone die fabrication, wherein the stainless steel die was duplicated in a low-expansion die stone (Fig. 2) using an acrylic custom tray with a $1.5 \mathrm{~mm}$ wax spacer and polyvinyl siloxane impression material. The stone die was accurately positioned and secured into the stainless steel former. Further the wax pattern fabrication was performed. Crown and bridge modeling wax was placed in the stainless steel

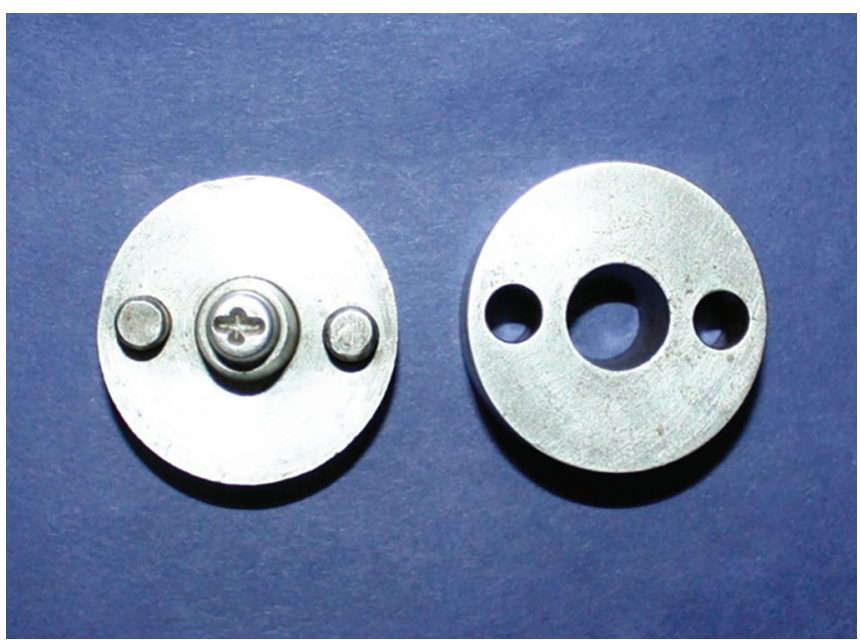

Fig. 1: Occlusal view of custom-made stainless steel die, stainless steel-former assembly

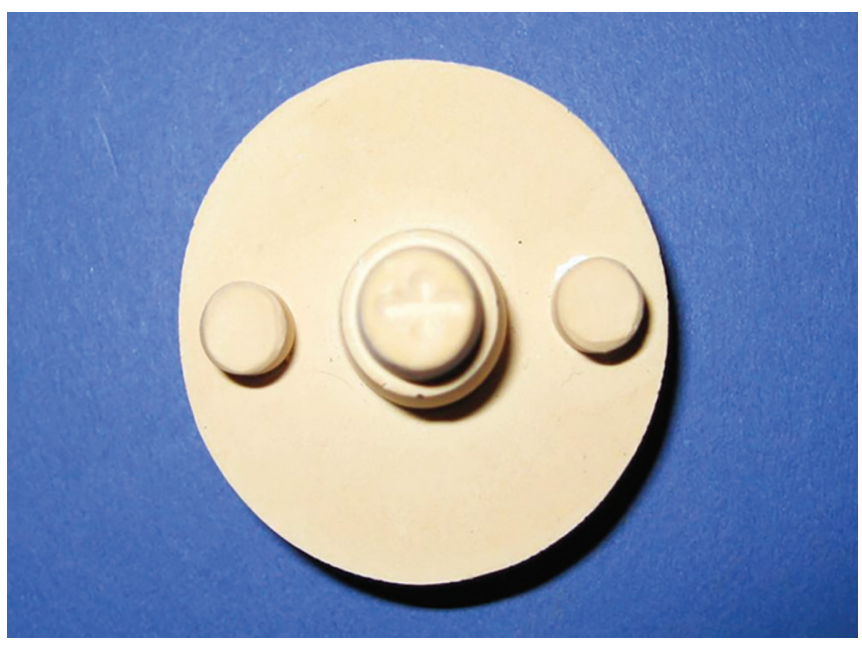

Fig. 2: Duplicated die of the stainless steel die in die stone 
Table 1: Study groups and their representation

\begin{tabular}{ll}
\hline Group & Representation \\
\hline Group A & Representing wax patterns \\
Group B & Representing castings with no ring liners \\
Group C & Representing castings with dry ring liners \\
Group D & Representing castings with wet ring liners \\
\hline
\end{tabular}

former and melted with a torch. The lubricated stone die was positioned in the steel former. The die-former assembly was held together for 1 minute and then immersed in room temperature water for 3 minutes. The die was then separated from the former and all excess wax was removed from the margin area before the wax pattern was removed from the stone die. The intaglio surface of each wax pattern was visually inspected under a magnification lens to ensure that it was smooth and free of defects. The wax pattern was then repositioned on its stone die and stored in a plastic container at room temperature for 48 hours. All wax patterns were uniform with a $1 \mathrm{~mm}$ thickness for all axial and occlusal walls. A total of 40 stone dies and wax patterns were fabricated. They were divided into the following four groups that consisted of 10 individual wax patterns and stone dies each (Table 1).

- Group A: Representing wax patterns

- Group B: Representing castings with no ring liners

- Group C: Representing castings with dry ring liners

- Group D: Representing castings with wet ring liners

Then the spruing of the 30 wax patterns (group B, group C, and group D) was done, followed by the metal ring investment procedures and ring liner placement. Further, the investment setting time (bench setting) of 1 hour was kept for the conventional wax elimination procedure that was based on the manufacturer's recommendations. The mean time interval required for the investment material to reach its maximum exothermic setting reaction temperature was determined using a digital thermometer. A three-stage wax elimination cycle was used for the conventional technique in accordance with the manufacturer's recommendations. One ring and muffle at a time were placed into the center of an electric furnace at room temperature.

All casting procedures were performed by the same operator using an induction centrifugal casting machine. Two new pellets of non-precious $(62.9 \% \mathrm{Ni}, 23.0 \% \mathrm{Cr}, 10 \% \mathrm{Mo})$ metal ceramic alloy were used for each casting. The castings were then cleaned with distilled water in an ultrasonic cleaner for 12 minutes, sprayed with an air-water syringe, and dried.

The outcome was measured as the amount of marginal discrepancy. Each casting was seated on its respective stone die under a constant load of $2 \mathrm{~kg}$ that was applied onto the occlusal surface for 10 seconds. Grooves on the occlusal surface of the die prevented the casting from rotating and ensured that the crown was seated at the same position as the wax pattern. Measurements were made using a digital optical microscope with an accuracy of $0.1 \mu \mathrm{m}$. The casting was seated onto the stone die and was clamped horizontally in position by the metallic jig fixture of the digital microscope with two-and-a-half turns of the thumbscrew. The measurements were made between the margin of the casting and the two reference marks scribed on the die stone that were separated at $180^{\circ}$ (Figs 3 and 4). All measurements were performed by the same operator without any knowledge of the identity of the testing groups.

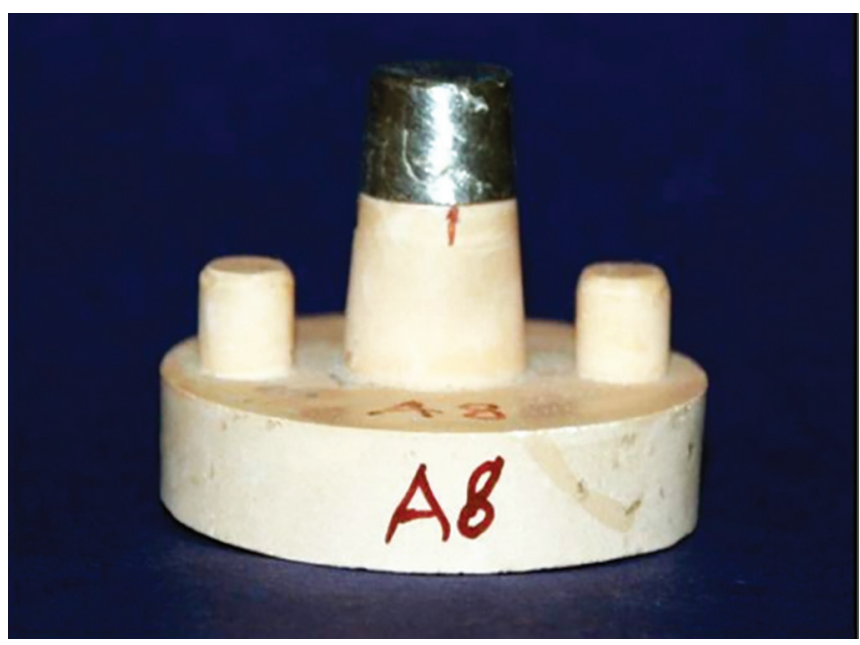

Fig. 3: Castings with dry ring liners with marking of $0^{\circ}$

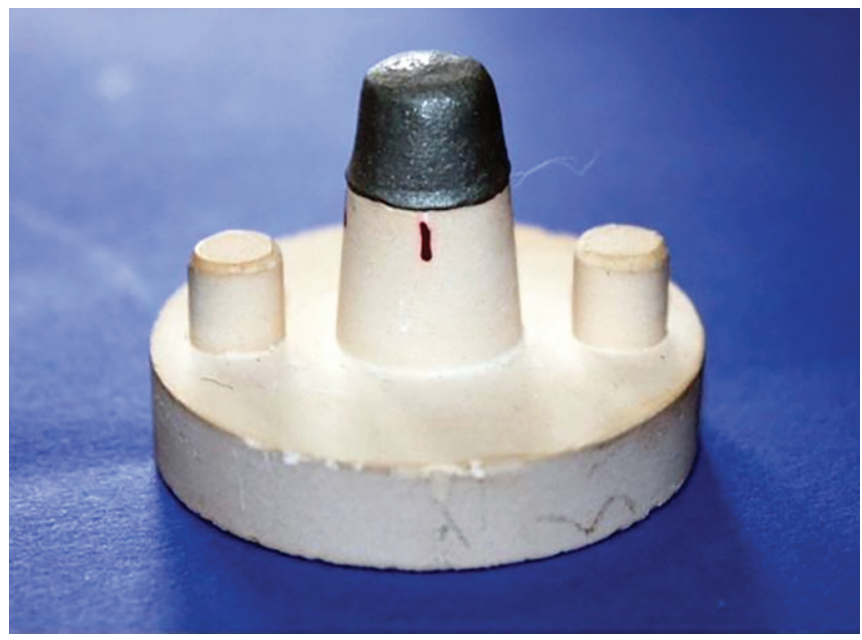

Fig. 4: Castings with dry ring liners with marking of $180^{\circ}$

\section{Statistical Analysis}

Data collected were entered in Microsoft Excel (2013), and statistical analysis was carried out by the usage of statistical product and service solutions (v.21.0). Descriptive analysis was performed to determine the mean marginal discrepancy values in all the groups keeping $95 \%$ confidence intervals and having a $p$-value statistically significant of less than 0.05 .

\section{Results}

In this study, a total of 40 stone dies and wax patterns were fabricated, which were divided into different groups based on their states (wet or dry) and based on the presence or absence of the liners. The mean marginal discrepancy was assessed in all the groups.

Our study results showed marginal discrepancy in all $30 \mathrm{Ni}-\mathrm{Cr}$ copings castings that used dry, wet, or no cellulose ring liners. The marginal discrepancy of the 10 wax patterns showed a mean value of $15.43 \mu \mathrm{m}$. The marginal discrepancy of the 10 cast copings that were made using casting with dry cellulose ring liners showed a mean value of $28.34 \mu \mathrm{m}$. The marginal discrepancy of the 10 cast copings that were made casting without using any ring liners showed a mean value of $30.81 \mu \mathrm{m}$. The marginal discrepancy of the 
Marginal Discrepancies with and without Using Ring Liners

Table 2: Mean vertical marginal discrepancies in different groups in the study samples

\begin{tabular}{|c|c|c|c|c|c|c|c|c|}
\hline \multirow[b]{2}{*}{ Sample } & \multicolumn{2}{|c|}{$\begin{array}{c}\text { Group A } \\
\text { Wax pattern }\end{array}$} & \multicolumn{2}{|c|}{$\begin{array}{c}\text { Group B } \\
\text { No ring liner }\end{array}$} & \multicolumn{2}{|c|}{$\begin{array}{c}\text { Group C } \\
\text { Dry ring liner }\end{array}$} & \multicolumn{2}{|c|}{$\begin{array}{c}\text { Group D } \\
\text { Wet ring liner }\end{array}$} \\
\hline & $0^{\circ}$ & $180^{\circ}$ & $0^{\circ}$ & $180^{\circ}$ & $0^{\circ}$ & $180^{\circ}$ & $0^{\circ}$ & $180^{\circ}$ \\
\hline 1 & 30 & 0 & 14.6 & 13.5 & 21 & 15.5 & 34.4 & 37 \\
\hline 2 & 19.9 & 0 & 40 & 7.3 & 34.2 & 21.7 & 27 & 24 \\
\hline 3 & 0 & 12 & 16.3 & 17 & 24.3 & 46.3 & 53.4 & 42.4 \\
\hline 4 & 14 & 0 & 19.6 & 20.3 & 24.8 & 31 & 61.7 & 80.7 \\
\hline 5 & 20 & 12 & 42 & 45.9 & 15.3 & 19.6 & 38.6 & 34.8 \\
\hline 6 & 24 & 22 & 43.6 & 31 & 39.8 & 26.5 & 26 & 24 \\
\hline 7 & 15 & 19 & 26.9 & 70 & 28 & 37.3 & 21.7 & 24.9 \\
\hline 8 & 18.5 & 20 & 24.4 & 19 & 46.4 & 36.3 & 48.1 & 68.1 \\
\hline 9 & 0 & 12 & 44.4 & 65.3 & 27.9 & 30.3 & 24.8 & 26.5 \\
\hline 10 & 12.9 & 13 & 36.3 & 39.8 & 21.7 & 29.3 & 21.7 & 29.3 \\
\hline Mean & 15.4 & 11 & 30.8 & 32.91 & 24.34 & 29.35 & 35.74 & 39.17 \\
\hline
\end{tabular}

10 cast copings obtained from casting using cellulose ring liner in a wet state showed a mean value of $35.74 \mu \mathrm{m}$ (Table 2).

The order of discrepancy values of marginal gap of the cast copings in the study is as follows:

- Minimum marginal gap-casting using cellulose ring liner in dry state $28.34 \mu \mathrm{m}$.

- Maximum marginal gap-casting using cellulose ring liner in wet state $35.74 \mu \mathrm{m}$.

The castings made using dry cellulose ring liners showed minimal marginal discrepancies compared with castings obtained from using wet cellulose ring liners or no ring liners.

\section{Discussion}

Our in vitro study assessed the varied marginal discrepancies that can occur with or without the use of cellulose ring liners in castings that would alter and affect the final fit of the restorations. The marginal integrity and fit of the crown margin are important for the long-term success of a cast restoration. ${ }^{13}$ The marginal fit is related to the clinical viability and longevity of the cast restoration. These factors are clinically relevant to periodontal health and the development of recurrent marginal caries. ${ }^{14}$ A well-fit cast alloy crown has sufficient axial tolerance to allow proper seating and its margin is congruent with the cavosurface line angle of the tooth preparation, as judged by visual and tactile examinations. The marginal fit of the castings relies on careful tooth preparation, accurate impressions, and precision casting.

Misfit castings can be classified by taking measurements at various points between the casting surface and the tooth. ${ }^{15}$ These measurements are taken at different locations that are geometrically related to each other and defined as the internal gap, marginal gap, vertical marginal discrepancy, horizontal marginal discrepancy, overextended margin, underextended margin, absolute marginal discrepancy, and the seating discrepancy. ${ }^{14}$

Several studies ${ }^{15,16}$ were conducted to evaluate the marginal defects of castings. Bjorn et al. ${ }^{2}$ discovered that $83 \%$ of gold and $74 \%$ of porcelain crown margins had approximal defects that were greater than $0.2 \mathrm{~mm}$. Schwartz ${ }^{3}$ showed that defective margins were a frequent cause (11.3\%) of unserviceable crowns and fixed partial dentures. It was reported by Davis $\mathrm{DR}^{5}$ that $30-40 \%$ of crown margins were inadequate, often due to poor marginal integrity. Recent studies ${ }^{17,18}$ continue to find high rates of marginal deficiencies and show that defective margins are responsible for over $10 \%$ of all failed units.

In our study, all of the aforementioned factors were considered during the fabrication and investing of the wax patterns to minimize the effects of wax distortion on the marginal discrepancies of the castings. Forty wax patterns were fabricated with an accurate steel counter die, which was placed on a die stone replica of the master steel die. The counter die was $1 \mathrm{~mm}$ larger than the master die, which provided a uniform space to pour the low-softening-temperature wax into to ensure that the wax patterns were uniform. The wax patterns were then uniformly distributed into four groups (10 per group) and cast using the previously described methodology. To determine the practicality of the previously described procedures, we designed a study to compare the marginal discrepancies associated with wet, dry, and absent cellulose ring liners using the metal ring casting technique and a conventional wax elimination procedure for 30 castings.

Davis ${ }^{4}$ described that wet asbestos liners provided additional water to the setting investment, which led to increased setting expansion while allowing for sufficient radial expansion by compressing the ring liner. This led to increased marginal gaps and thus altered the fittings. Similar results were noted in our study, wherein the wet ring liners demonstrated more discrepancies as compared to no liners/dry ring liners. Another study ${ }^{19}$ described the frequent use of asbestos liners in investment metal casting for dental laboratory work that was associated with risks asbestos to the human body have led to the development and marketing of casting liners without asbestos by many manufacturers. These are the so-called non-asbestos liners.

Earnshaw ${ }^{9}$ showed that cellulose paper (used wet) and ceramic paper (used dry) are replacing asbestos paper as the cushioning ring liner in dental castings. Wet cellulose liners have an effect that is similar to that of traditional wet asbestos liners. Dry ceramic liners are associated with a much lower investment expansion, which could be an advantage for investments that have an increased measured expansion. These results are concurrent with the results obtained in our study.

Another study conducted by Keyf and Erman ${ }^{1}$ noted that the carcinogenic potential of asbestos has led to the development of casting ring liners made from non-asbestos materials, such as 
cellulose paper, to replace asbestos paper. They reported that dry liners are easy to control and may improve the fit of the cast to the die, but is generally accepted that any of these materials can produce consistently acceptable castings when paired with the appropriate techniques.

In the present study, 30 castings were fabricated using a base metal alloy (Heraenium $\mathrm{S}, \mathrm{Ni}-\mathrm{Cr}$ dental casting alloy, Heraus Kulzer, Germany) that consisted of nickel $62.9 \%$, chromium $23.0 \%$, molybdenum $10.0 \%$, silica $2.0 \%$, iron $1.5 \%$, and cerium $<1 \%$. Base metal alloys, including cobalt-chromium (Co-Cr) and nickelchromium ( $\mathrm{Ni}-\mathrm{Cr}$ ), have been widely used in dentistry to fabricate fixed and removable partial denture frameworks since being introduced in 1929. These alloys have increasingly replaced gold and other precious alloys due to their superior mechanical properties and reduced cost. The primary physical and chemical properties of these base metal alloys include a lower density than gold alloys, which is a particularly useful feature for fabricating less bulky prostheses, and a modulus of elasticity that is nearly twice that of gold alloys, which is useful for fixed and removable partial dentures as it has the advantage of maintaining rigidity with less bulk. These properties allow for improved esthetics and physiological contouring and can be used to develop a suitable occlusion with less tooth structure reduction. More recently, improvements in alloy compositions and the development of new manufacturing techniques have optimized the use of these alloys, which made them suitable for the present study.

A literature review demonstrated the controversy over defining a marginal fit value that is clinically acceptable for cast restorations. Some authors reported that $30 \mu \mathrm{m}$ is a clinically acceptable marginal gap. However, Schwartz ${ }^{3}$ defined a clinically acceptable marginal misfit that was between 10 and $160 \mu \mathrm{m}$. Leong et al. ${ }^{20}$ considered $120 \mu \mathrm{m}$ to be acceptable, while Samet et al. ${ }^{21}$ and Contreras et al. ${ }^{22}$ agreed that a value below $100 \mu \mathrm{m}$ was the most appropriate value for adequate restoration longevity. Another study reviewed expert opinions on acceptable margin openings and reported a range between 32 and $230 \mu \mathrm{m}$ with disagreements not only between subjects but also within subjects.

In this study, the appropriate and adequate measures were used to control the technicality in the die stone fabrication and to minimize errors while casting procedure. All these contribute to the strengths of our study. But, in our study, the marginal discrepancies were measured at two predetermined points ( 0 and 180). Increasing the number of reference points to measure the marginal gaps for each coping could provide more conclusive results. Despite these limitations, this in vitro study suggests that the marginal fit of cast copings made with two different methods of pattern forming was within the range of clinically acceptable values for adequate longevity of restorations. The results from this study should be extrapolated and expanded in further studies by evaluating different pattern-forming materials and techniques and identifying factors that could improve the marginal fit of cast restorations. Future studies of cast restorations in intraoral conditions and the specific factors that affect them are also needed.

\section{Conclusion}

In this in vitro study, the cast restorations made from casts with dry cellulose ring liners showed minimal vertical marginal discrepancies when compared with cast restorations obtained from casts with wet cellulose ring liners or no ring liners. Future studies on casting with different casting ring liners and different ring liner states should be carried out using a range of parameters to confirm our findings and to improve the estimates of marginal discrepancies.

\section{References}

1. KeyfF, Erman G. Full-crown castings made with water-saturated and dry casting ring liners. J Biomater Appl 2000;14(4):399-408. DOI: 10.1177/088532820001400406.

2. Bjorn AL, Bjorn H, Grkovic B. Marginal fit of restorations and its relation to periodontal bone level. II. Crowns. Odontol Revy 1970;21(3): 337-346. PMID: 5275036.

3. Schwartz IS. A review of methods and techniques to improve the fit of cast restorations. J Prosthet Dent 1986;56(3):279-283. DOI: 10.1016/0022-3913(86)90003-x.

4. Davis DR. Release of asbestos fibers during casting ring liner manipulation. J Prosthet Dent 1995;74(3):294-298. DOI: 10.1016/ s0022-3913(05)80137-4.

5. Davis DR. Effect of wet and dry cellulose ring liners on setting expansion and compressive strength of a gypsum-bonded investment. J Prosthet Dent 1996;76(5):519-523. DOI: 10.1016/s00223913(96)90011-6.

6. Ross MA. The "asbestos" minerals: definitions, descriptions, modes of formation, physical and chemical properties, and health risks to the mining community. Nat Bur Std 1978;506:29-63.

7. Ross MA. The geologic occurrences and health hazards of amphibole and serpentine asbestos. In:Veblen DR, editor. Reviews in mineralogy. Mineralogical Society of America; 1981. pp 279-323.

8. Taylor NO, Paffenbarger GC. A survey of current inlay casting techniques. J Am Dent Assoc 1930;17(11):2058-2081. DOI: 10.14219/ jada.archive.1930.0311.

9. Earnshaw R. The effect of casting ring liners on the potential expansion of a gypsmn bonded investment. J Dent Res 1988;67(11): 1366-1370. DOI: 10.1177/00220345880670110101.

10. Haralur SB, Hamdi OA, Al-Shahrani AA, et al. The effect of casting ring liner length and prewetting on the marginal adaptation and dimensional accuracy of full crown castings. J Int Soc Prevent Community Dent 2017;7(1):52-57. DOI: 10.4103/jispcd. JISPCD_483_16.

11. Hickel R, Peschke A, Tyas M, et al. FDI World Dental Federation: clinical criteria for the evaluation of direct and indirect restorations-update and clinical examples. Clin Oral Investig 2010;14(4):349-366. DOI: 10.1007/s00784-010-0432-8.

12. Krithikadatta J, Gopikrishna V, Datta M. CRIS guidelines (checklist for reporting in-vitro studies): a concept note on the need for standardized guidelines for improving quality and transparency in reporting in-vitro studies in experimental dental research. J Conserv Dent 2014;17(4):301-304. DOI: 10.4103/0972-0707.136338.

13. Neppelenbroek KH. The clinical challenge of achieving marginal adaptation in direct and indirect restorations. J Appl Oral Sci 2015;23(5):448-449. DOI: 10.1590/1678-77572015ed005.

14. Rong Y, Luo X, Zhang Z, et al. Occupational exposure to asbestos and cardiovascular related diseases: a meta-analysis. Prev Med Rep 2015;2:920-926. DOI: 10.1016/j.pmedr.2015.10.005.

15. Morey EF. Dimensional accuracy of small gold alloy castings. Part 3. Gypsum bonded investment expansion. Aust Dent J 1992;37(2):43-54. DOI: 10.1111/j.1834-7819.1992.tb03043.x.

16. Hutton JE, Marshall GW. Expansion of phosphate-bonded investments: Part II-Thermal expansion. J Prosthet Dent 1995;73(2):127-131. DOI: 10.1016/s0022-3913(05)80150-7.

17. Anwar M, Tripathi A, Kar SK, et al. Effect of PFM firing cycles on the mechanical properties, phase composition, and microstructure of nickel-chromium alloy. J Prosthodont 2015;24(8):634-641. DOI: 10.1111/jopr.12328.

18. Kalavathi M, Sachin B, Prasanna BG, et al. Marginal accuracy of castings fabricated with ringless casting investment system and metal ring 
casting investment system: a comparative study. J Contemp Dent Pract 2016;17(2):165-170. DOI: 10.5005/jp-journals-10024-1821.

19. Boitelle $P$, Mawussi $B$, Tapie $L$, et al. A systematic review of CAD/CAM fit restoration evaluations. J Oral Rehabil 2014;41(11):853-874. DOI: 10.1111/joor.12205.

20. Leong D, Chai J, Eugene L, et al. Marginal fit of machine-milled titanium and cast titanium single crowns. Int J Prosthodont 1994;7(5):440-447. PMID: 7802912.
21. Samet N, Resheff B, Gelbard S, et al. A CAD/CAM system for the production of metal copings for porcelain-fused-to-metal restorations. J Prosthet Dent 1995;73(5):457-463. DOI: 10.1016/s00223913(05)80075-7.

22. Contreras $\mathrm{E}$, Henriques $\mathrm{G}$, Giolo $\mathrm{S}$, et al. Fit of cast commercially pure titanium and Ti-6Al-4V alloy crowns before and after marginal refinement by electrical discharge machining. J Prosthet Dent 2002;88(5):467-472. DOI: 10.1067/mpr.2002.128954. 